\title{
$\beta$ Cephei and Slowly Pulsating B stars as targets for BRITE-Constellation
}

\author{
G. Handler
}

Institut für Astronomie, Türkenschanzstrasse 17, 1180 Vienna, Austria

\begin{abstract}
We discuss the potential of BRITE-Constellation for asteroseismology of main sequence B-type pulsators. We briefly review previous asteroseismic work on these oscillators and point out the importance of these stars for astrophysics in general. We find that BRITE-Constellation is ideally suited for studying B-type pulsators, and we identify several interesting regions in the sky that may be observed.
\end{abstract}

\section{Introduction}

This workshop has seen many interesting presentations of proposed targets that were better understood through observational data from BRITE-Constellation. Obviously, realizing the potential of a new instrument for the study of the object one is interested in, is probably any scientist's initial reaction. However, in only a few cases it was stated what the general impact of the proposed studies on astrophysics would be. Perhaps it is worth considering a different viewpoint.

During the Vienna Workshop on the Future of Asteroseismology in September 2006 (Handler \& Houdek 2007), Kjeldsen (2007) made an important statement. "We promised to do asteroseismology but if you look on where we really changed the understanding of the evolution of the universe, we haven't gone where we promised yet, but we hope that space missions can do that. We have to make sure that the whole community can use all these space data. If we make another effort to spread all these data to students around the whole world, they will all learn the way seismologists study stars. Then we will have a much better chance to fund the next mission."

In other words, we first have to see how we can most efficiently use the resources we have been given, to obtain a scientific output that has the maximum possible impact on astrophysics in general, and to ensure the existence of our scientific field in the future. Only after we have found out what we can do for BRITE-Constellation to have as much scientific impact as possible, we should think about what BRITE-Constellation can do for us. 


\section{$\beta$ Cephei and SPB stars}

These two types of pulsating variables are main-sequence objects of spectral type B. Both have relatively simple interior structures, with convective cores and (mostly) radiative envelopes, but their pulsational behaviour is clearly distinct. Whereas the $\beta$ Cephei stars pulsate in pressure and gravity modes of low radial order, with corresponding periods of 2 - 8 hours, the SPB stars exhibit gravity modes of high radial order in the period range of $0.5-6$ days. The $\beta$ Cephei stars are the most massive known main sequence pulsators $\left(9<M<17 M_{\odot}\right.$, Stankov \& Handler 2005) and they are progenitors of supernovae of type II. The locations of both types of variables in the HR diagram are shown schematically in Fig. 1.

\section{Asteroseismic results for $\beta$ Cephei stars}

Asteroseismology is the study of the interior of (non-radially) pulsating stars by means of their normal mode spectrum. This technique is analogous to the determination of the Earth's inner structure using earthquakes: we use stellar pulsations as "starquakes" or, more scientifically, we use stellar pulsation modes as seismic waves.

Many types of pulsating stars have been investigated with seismic methods, but real insights into their interior structures have rarely been obtained so far. The only exceptions are the Sun and some solar-like pulsators, pulsating white dwarf stars and the $\beta$ Cephei stars. It is out of the scope of this paper to give an overview of the prospects and problems of asteroseismology, which is why we refer to Handler (2006) for a summary of these issues.

Why has asteroseismology been successful for the $\beta$ Cephei stars? The answer seems trivial: they are simple enough that the pulsation modes can be identified, yet complicated enough to reveal effects of interior structure. At the time of this writing, there are four stars that have unique mode identifications and sufficiently rich pulsation spectra for seismic modelling, HD 129929 (Aerts et al. 2004), $\nu$ Eridani (e.g. Handler et al. 2004, Pamyatnykh et al. 2004, Jerzykiewicz et al. 2005), $\theta$ Ophiuchi (Handler et al. 2005, Briquet et al. 2005) and 12 Lacertae (Handler et al. 2006, Desmet et al., in preparation).

The seismic results obtained from these studies can be summarized as follows: the presence of differential interior rotation and the need of a small convective overshooting parameter $\left(\alpha_{\mathrm{ov}}<0.15\right)$ have been proven for HD 129929 and $\nu$ Eri. For the latter star, the mean interior metallicity has been determined as well. For the stars $\theta \mathrm{Oph}$ and $12 \mathrm{Lac}$, which rotate somewhat faster than HD 129929 and $\nu$ Eri, the interaction between pulsation and rotation can be studied. 


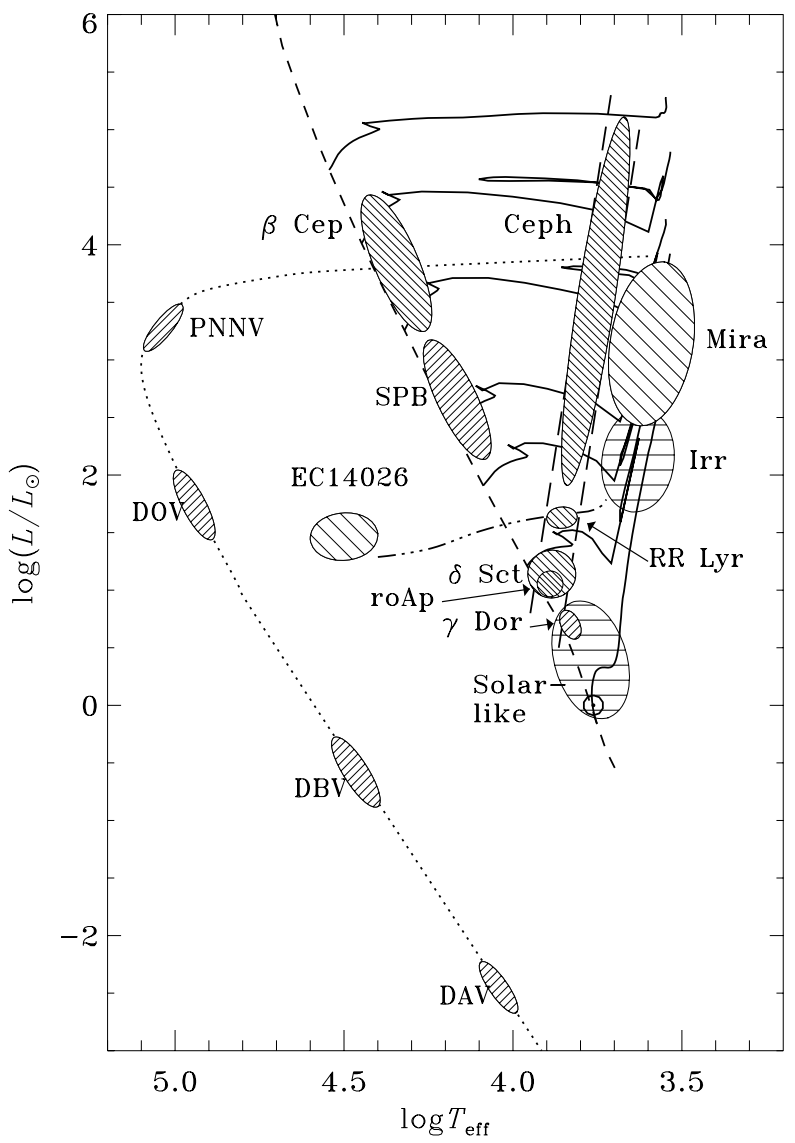

Figure 1: A theoretical HR diagram containing the locations of most of the known pulsating variables. The $\beta$ Cephei and SPB stars can be found near the high-luminosity end of the main sequence. Reproduced with permission from J. Christensen-Dalsgaard.

However, some problems with the theoretical description of pulsational driving still remain, as the observed frequency spectra have a larger range than predicted. The obvious prime suspects in this context are the stellar opacities (e.g., Pamyatnykh \& Ziomek 2007), or possible effects of diffusion. 
Asteroseismology of SPB stars

Because these stars pulsate in high-order gravity modes, they may facilitate the application of asymptotic theory, greatly enhancing the prospects of mode identification and stellar modelling. This potential asset is also a difficulty because it follows from the long pulsation periods that only few pulsation modes have so far been detected (e.g., De Cat \& Aerts 2002). In addition, the theoretical treatment of pulsation becomes difficult even at moderate rotation rates, as the pulsation and rotation periods are of the same order and a treatment of rotation as a perturbance is no longer possible. However, recent MOST satellite data may have revealed much larger numbers of pulsation frequencies for some SPB stars (e.g., Aerts et al. 2006), although data sets with a longer time base would be desirable for purposes of confirmation.

Another interesting possibility is the co-existence of stars that have both SPB star-like gravity modes and shorter-period $\beta$ Cephei-type pulsation modes excited. Evidence is mounting that several such stars exist (e.g., De Cat et al. 2007 and references therein, Pigulski \& Pojmanski 2007). It will be of tremendous help for the seismic application of the SPB star pulsations if some of these "hybrid" stars have pulsation spectra sufficiently rich to utilize both sets of modes.

\section{BRITE-Constellation and B-type main sequence pulsators}

A check of the latest catalogues of $\beta$ Cep and SPB stars (Stankov \& Handler 2005, De Cat 2002) for variables with $V<4$ reveals a large number of possible candidates observable by BRITE-Constellation. We find 17 known and 12 candidate $\beta$ Cep stars under these constraints, with another 35 stars inside the $\beta$ Cep instability strip. In addition, there are three known SPB pulsators plus three more candidates among these brightest stars in the sky.

Still, this is not the whole story. As both types of stars have short main sequence lifetimes, they are all young objects and therefore occur in stellar clusters and associations. Investigating the distribution of these stars in the sky, and comparing it with the field of view of BRITE-Constellation, several interesting $25 \times 25$-degree regions in the sky can be located; they are summarized in Table 1.

These seven fields contain a total of eleven known pulsators (some of which have already been studied from the ground, which means they have pulsational mode identifications) plus 42 other possible targets. We note that O-type stars in the BRITE-Constellation fields are also interesting from the asteroseismic point of view because theory predicts oscillations in such stars (Pamyatnykh 1999), but observations have rarely detected them. 
Table 1: $25 \times 25$-degree regions in the sky containing large numbers of $\beta$ Cep and SPB stars and candidates $(V<4)$

\begin{tabular}{ll}
\hline Region & Stellar content \\
\hline Cen/Cru/Mus & $1 \beta$ Cep star, $9 \beta$ Cep candidates, 1 SPB candidate \\
Cen/Lup & $2 \beta$ Cep stars, $5 \beta$ Cep candidates, 2 SPB candidates \\
Upper Sco & $1 \beta$ Cep star, $7 \beta$ Cep candidates, 1 O-type star \\
Orion & $1 \beta$ Cep star, $3 \beta$ Cep candidates, 5 O-type stars \\
Centaurus & $2 \beta$ Cep stars, $5 \beta$ Cep candidates \\
Lower Sco/Oph & $3 \beta$ Cep stars, $2 \beta$ Cep candidates \\
CMa OB1 & $1 \beta$ Cep star, $2 \beta$ Cep candidates \\
\hline
\end{tabular}

How can the science output of BRITE-Constellation benefit from observing these stars? It will be possible to perform asteroseismology of supernova progenitor stars; supernovae largely determine the ecology of the universe. We also expect a general improvement in our understanding of stellar structure and evolution, such as angular momentum transport (i.e. differential interior rotation) and the size of the convective cores of massive stars, and we expect constraints on stellar opacities and diffusion from studies of mode excitation.

Why is BRITE-Constellation not only suitable, but even required for studying massive main sequence pulsators? It simply can perform efficient observations of regions densely populated with bright stars. If we compare the quantity and quality of the data to be delivered by BRITE-Constellation to that of classical ground-based observations of bright stars, we find that the efficiency of the space mission is a factor of about 100 higher. To put it short: the future of asteroseismology is BRITE!

Acknowledgments. GH is supported by the Austrian Fonds zur Förderung der wissenschaftlichen Forschung under grant P18339-N08.

\section{References}

Aerts, C., Waelkens, C., Daszyńska-Daszkiewicz, J., et al., 2004, A\&A 415, 241

Aerts, C., de Cat, P., Kuschnig, R., et al., 2006, ApJ 642, L165

Briquet, M., Lefever, K., Uytterhoeven, K., et al. 2005, MNRAS 362, 619

De Cat, P., 2002, http://www.ster.kuleuven.ac.be/ peter/Bstars/

De Cat, P., Aerts C. 2002, A\&A 393, 965

De Cat, P., Briquet, M., Aerts, C., et al. 2007, A\&A 463, 243

Handler, G., 2006, CoAst 147, 31

Handler, G., Houdek, G. 2007, CoAst Vol. 150 
Handler, G., Shobbrook, R.R., Jerzykiewicz, M., et al. 2004, MNRAS 347, 454

Handler, G., Shobbrook, R.R., Mokgwetsi, T. 2005, MNRAS 362, 612

Handler, G., Jerzykiewicz, M., Rodr' Iguez, E., et al. 2006, MNRAS 365, 327

Jerzykiewicz, M., Handler, G., Shobbrook, R.R., et al. 2005, MNRAS 360, 619

Kjeldsen, H. 2007, CoAst 150, 375

Pamyatnykh, A.A. 1999, Acta Astron. 49, 119

Pamyatnykh, A.A., Ziomek, W. 2007, CoAst 150, 207

Pamyatnykh, A.A., Handler, G., Dziembowski, W.A. 2004, MNRAS 350, 1022

Pigulski, A., Pojmanski, G. 2007, A\&A, in press

Stankov, A., Handler, G. 2005, ApJS 158, 193 